\title{
Synthesis and Characterization of Ferrofluid-Chitosan-Au Nanoparticles as Brachytherapy Agent Candidate
}

\author{
Muflikhah $^{1^{*}}$, Ahmad Marzuki Ramadhan ${ }^{2}$, Maria Christina Prihatiningsih ${ }^{2}$, \\ Mujamilah ${ }^{1}$, and Aloma Karo Karo ${ }^{1}$ \\ ${ }^{1}$ Center for Science and Technology of Advanced Materials, National Nuclear Energy Agency of Indonesia, \\ Kawasan Puspiptek Serpong, Banten 15314, Indonesia \\ ${ }^{2}$ Polytechnic Institute of Nuclear Technology, National Nuclear Energy Agency of Indonesia, \\ Jl. Babarsari POB 6101 Ykbb, Yogyakarta 55281, Indonesia
}

\section{* Corresponding author:}

tel: $+62-85640680600$

email:muflikhah@batan.go.id

Received: December 13, 2020

Accepted: May 24, 2021

DOI: $10.22146 / \mathrm{ijc} .62191$

\begin{abstract}
Brachytherapy is expected to be a solution to the side effect of other cancer therapy methods. This study aims to synthesize ferrofluids (FF)-Chitosan-Au (so-called cold synthesis) as the initial step before using ${ }^{198} \mathrm{Au}$ that is expected to be a targeted and controllable brachytherapy agent. In this research, the preparation of FF-Chitosan was done by the co-precipitation method. Furthermore, FF-Chitosan-Au was produced via self-assembly by the adsorption method. The adsorption followed the Langmuir model with a maximum capacity of $30.24 \mathrm{mg} \mathrm{Au/g} \mathrm{FF-Chitosan.} \mathrm{The} \mathrm{X-Ray} \mathrm{Diffractometion}$ (XRD) of FF-Chitosan-Au confirms the existence of Au. Particle Size Analyzer (PSA) indicates FF-Chitosan-Au has an average size of $82.93 \mathrm{~nm}$ with a polydispersity index of 0.175. Morphological and distribution analysis of nanoparticles using Scanning Electron Microscope (SEM) shows that nanoparticles have a homogenous spherical shape. Vibrating Sample Magnetometer (VSM) measurement confirms the superparamagnetic properties of FF-Chitosan and FF-Chitosan-Au with a saturated magnetization of 80.48 and $74.52 \mathrm{emu} / \mathrm{g}$, respectively. The overall results are associated with biomedical requirements, such as high saturation magnetization and good polydispersity. The synthesis can also be applied to produce FF-Chitosan- ${ }^{198} \mathrm{Au}$ that has great potential as a brachytherapy agent, which will reduce the nuclear waste and potential danger of radiation received by workers during synthesis.
\end{abstract}

Keywords: ferrofluids; magnetic nanoparticle; adsorption; chitosan-gold, brachytherapy

\section{- INTRODUCTION}

Research of nanomaterials by assembling some nanomaterials components becomes interesting due to having versatile physical and biological properties that have a wide range of applications such as in catalysis and targeting therapeutic drugs [1-2]. For example, nanoparticle magnetic is one of the favorite materials combined with other materials by surface modification, especially in biomedical applications [3]. This modification aims to make magnetic nanoparticles (MNP) being compatible with the bio-system [4].

Recently, research on targeted therapy agents has become the most promising cancer treatment option [5].
Extensive studies on chemically modified polymeric MNP have been done because of their capability of carrying a wide range of drugs to tumor sites [5]. However, for biomedical applications, MNP-mostly magnetite must have high saturation magnetization, stability, biocompatibility, and interactive functions at the surface [6]. On the other hand, MNP has hydrophobic surfaces that tend to agglomerate due to strong magnetic dipole-dipole interaction, which causes different behavior [7]. Moreover, the naked MNP have high chemical activity and are easily oxidized in the air (especially magnetite), resulting in loss of magnetism and dispersibility [8-9]. Therefore, the surface coating 
should be done as effective protection strategies to keep the stability of MNP. The MNP surfaces were generally coated by organic molecules (surfactants, polymers, and biomolecules) or an inorganic layer (silica, metal, nonmetal elementary substance, metal oxide, or metal sulfide). These coating strategies protect and stabilize the MNP and play a crucial role in improving MNPs specificity; hence, the higher the targeting specificity, the more selective target cancer cells will be achieved [10-12].

Development of MNP coated by organic molecule becomes important in biomedical application due to its wide range of functions such as targeted drug delivery and radiotherapy and diagnostic agents [13]. Chitosan is a polyamine molecule with more advantages as an MNP coating agent than a neutral polymer such as polyethylene glycol (PEG) because of its ability to secondary functionalization [14-15]. Chitosan protects and stabilizes MNP agents and establishes electrostatic interactions with the negatively charged cell membrane in the weakly acidic microenvironment of tumors ( $\mathrm{pH}$ 6.8-7.2) [14-15]. In addition, Low Molecular Weight (LMW) chitosan as an MNP coating agent increases the hydrophilicity of the polymer and further improves its protective effect at neutral $\mathrm{pH}[16]$.

A variety of molecules or elements are able to bind with amine functional groups at chitosan. Gold is a noble metal that widely used in various fields, including health. Over the last decade, researchers have made many efforts to use gold nanoparticles for cancer treatment [4-5]. Gold has become an important material in nanomedicine due to its stability and ease of functionalization [15]. Gold nanoparticles (AuNPs) also have extraordinary tumor retention capabilities because of their natural affinity to leaky tumor vasculature, manifested in tumor angiogenesis [16]. Many research uses gold nanoparticles and their activated phase $\left({ }^{198} \mathrm{Au}\right)$ as therapeutic agents by different mechanisms, including thermal therapy, photodynamic therapy, and radiotherapy [14,17]. Gold nanoparticles are also extensively studied in the range of medical diagnostic and therapeutic applications (as theranostic agents) $[14,18]$. In order to reach the tumor target and avoiding its toxicity, gold combine with other molecules such as chitosan and magnetic material. Iron oxide/gold hybrid coated with chitosan has been studied for diagnostic and targeted drug delivery (theranostic agent) [18]. Pati and co-workers have synthesized chitosan functionalized iron oxide-Au core-shell for targeted drug delivery (TDD) [2]. Irradiated gold $\left({ }^{198} \mathrm{Au}\right)$ combined with poly(amidoamine) (PAMAM) for brachytherapy or internal radiotherapy has been inspected by Ritawidya and co-workers [17]. However, synthesis method of composite Au-iron oxide-chitosan that allows for activated $\mathrm{Au}$ has not been proposed yet. Many research of Au-iron oxide-chitosan added $\mathrm{Au}$ in the first step (before coated with chitosan). This method is quite difficult and not safe when using Au in activated phase. In this research, a more simple synthesis method of Au-iron oxide-chitosan nanocomposite has been studied which allows the using of irradiated $\mathrm{Au}$. The iron oxide-chitosan functionalized with $\mathrm{Au}$ in the final step by self-assembly. This method has benefit to reduce the chance of radioactive waste and the potential danger of radiation received by workers when using irradiated gold.

Synthesis of $\mathrm{Au}$-iron oxide-chitosan nanoparticles has been done in two steps. First, magnetite as a magnetic source that will play a role as a controllable therapy agent has been synthesized in chitosan solution to form Ferrofluid-Chitosan (FF-Chitosan) with sodium tripolyphosphate (Na-TPP) as a cross-linker [19]. Then, FF-Chitosan is functionalized with Au by self-assembly through the adsorption method in a batch system [13]. In this research, chloroauric acid tetrahydrate has been used as an Au source, then adsorbed by FF-Chitosan through electrostatic interaction. The influence of the initial $\mathrm{pH}$ of the $\mathrm{Au}$ was investigated to get the optimum $\mathrm{pH}$ of FF-Chitosan-Au synthesis. Langmuir and Freundlich isotherms were used to evaluate the equilibrium adsorption data [13]. This synthesis route will be the reference when using $\mathrm{Au}$ in the active phase $\left({ }^{198} \mathrm{Au}\right)$.

\section{- EXPERIMENTAL SECTION}

\section{Materials}

Chemicals used for the synthesis of FF-Chitosan$\mathrm{Au}$ were $\mathrm{FeCl}_{2} \cdot 4 \mathrm{H}_{2} \mathrm{O}$ and $\mathrm{FeCl}_{3} \cdot 6 \mathrm{H}_{2} \mathrm{O}$ from Sigma 
Aldrich as precursors for ferrofluid, chitosan with Low Molecular Weight (Sigma Aldrich), acetic acid (analytical grade, from Merck), sodium tripolyphosphate (Na-TPP) as a cross-linking agent, ammonium hydroxide $\left(\mathrm{NH}_{4} \mathrm{OH}\right)$ $32 \%$, distilled water with a resistivity of $18.2 \mathrm{M} \Omega \mathrm{cm}$, chloroauric acid tetrahydrate $\left(\mathrm{HAuCl}_{4}\right)$ from Sigma Aldrich. All chemicals in this study were used without further purification.

\section{Instrumentation}

Morphology and elemental analysis of nanoparticles Ferrofluid-Chitosan and Ferrofluid-Chitosan-Au were subjected to SEM-EDAX (JEOL-JED-2300). The estimation of the particle size has been done using DLS analysis (Malvern). XRD (PANalytical) was used for structural analysis. FTIR spectra of chitosan, FFChitosan, and FF-Chitosan-Au were recorded with Infrared Transmission Spectroscopy ( $\mathrm{KBr}$ module) in the range of $4000-400 \mathrm{~cm}^{-1}$ on FTIR-Bruker Tensor 27. The magnetization behavior of FF-Chitosan and FF-Chitosan$\mathrm{Au}$ were subjected to VSM (OXFORD 1.2H). Au concentrations in the supernatant (un-bounded $\mathrm{Au}$ ) were calculated using a Flammable Atomic Absorption Spectrophotometer (Perkin Elmer AAS Analyst 400).

\section{Procedure}

\section{Synthesis of Ferrofluid-Chitosan}

Ferrofluid-Chitosan (FF-Chitosan) nanoparticles were in-situ synthesized by co-precipitation method as described by Unsoy et al., with some modifications [20]. Ferrous chloride salts, $\mathrm{Fe}(\mathrm{II})$ and $\mathrm{Fe}(\mathrm{III})$, were dissolved in chitosan solution. TPP was used as a cross-linker. The synthesis has been conducted at room temperature by adding a total of $3.40 \mathrm{~g}$ of $\mathrm{FeCl}_{3} \cdot 6 \mathrm{H}_{2} \mathrm{O}$ and $2.68 \mathrm{~g}$ of $\mathrm{FeCl}_{2} \cdot 4 \mathrm{H}_{2} \mathrm{O}$ (mol ratio of $\mathrm{Fe}(\mathrm{II})$ and $\mathrm{Fe}(\mathrm{III})$ is 1:1), in $30 \mathrm{~mL}$ of $0.1 \% \mathrm{w} / \mathrm{v}$ chitosan in $1 \%$ acetic acid solution. Furthermore, the mixture of $\mathrm{Fe}(\mathrm{III})$ and $\mathrm{Fe}(\mathrm{II})$ chloride salts in chitosan solution was dispersed with ultrasound irradiation for $1 \mathrm{~min}$. Sodium-TPP $(0.00773 \mathrm{~g}$ dissolved in $10 \mathrm{~mL}$ DI-water) was mixed with $30 \mathrm{~mL}$ of $32 \%$ $\mathrm{NH}_{4} \mathrm{OH}$ and then added vigorously to the Fe-chitosan solution under ultrasonic irradiation for $5 \mathrm{~min}$. The resulting black-colored solution was then stirred using a mechanical mixer for an hour. The colloidal chitosan- coated $\mathrm{Fe}_{3} \mathrm{O}_{4}$ nanoparticles were extensively washed with deionized water and separated by magnetic decantation several times to remove the impurities. The solids were then added with deionized water and got additional ultrasound irradiation for $5 \mathrm{~min}$, then centrifuged for $30 \mathrm{~min}$ at $12000 \mathrm{rpm}$. The supernatant containing magnetic nanoparticles $\mathrm{Fe}_{3} \mathrm{O}_{4}$-chitosan was stored in the refrigerator.

\section{Synthesis of Ferrofluid-Chitosan-Au}

The synthesis of FF-Chitosan-Au has been carried out by the adsorption method in a batch system. FFChitosan were act as adsorbent and $\mathrm{HAuCl}_{4}$ as adsorbate. All the synthesis procedures were done at room temperature. In order to get the optimum condition, synthesis has been done in various $\mathrm{pH}$ of $\mathrm{Au}$ solution (1 to 6). Variation of FF-Chitosan amount (5, 10,15 , and $20 \mathrm{mg}$ ) and concentration of the gold solution $(40,60,80,100$, and $120 \mathrm{ppm})$ have been done at optimum $\mathrm{pH}$. In this study, the volume of Au solution used was $5 \mathrm{~mL}$ for each batch, and after the FF-Chitosan addition, the mixtures were shaken for $2 \mathrm{~h}$. Then, the adsorbent and supernatant were separated with an external magnet and centrifuge. The concentration of $\mathrm{Au}$ that remains in the supernatant was analyzed with Flammable Atomic Absorption Spectroscopy (FAAS). The resulting data of synthesis FF-Chitosan-Au in various gold solution concentrations were evaluated with Langmuir and Freundlich equations to determine the adsorption capacity.

\section{- RESULTS AND DISCUSSION}

This research is the initial research in the manufacture of FF-Chitosan-Au(198), which will be applied as a radiation source agent in brachytherapy. It is crucial to determine the optimum condition of synthesis using non-activated gold. This adsorption method (which $\mathrm{Au}$ takes place in the last step of synthesis) aims to reduce radioactive waste and the potential danger of radiation that workers will receive when synthesis using $\mathrm{Au}(198)$.

FF-Chitosan-Au has been synthesis in two steps. After FF-Chitosan has been obtained via coprecipitation, it was then bound to gold $\mathrm{Au}$ with 
adsorption method in a batch system. Magnetite nanoparticles (MNP) in ferrofluid form have been obtained in situ in chitosan solution with TPP as a crosslinker. Schematic interaction between MNP (ferrofluid) and chitosan with crosslinked TPP as a synthesis product is shown in Fig. 1. The amino groups in the chitosan have a positive charge as a result of protonation in an acidic medium that able to bond with the negative charge of hydroxyl groups of magnetite via electrostatic interaction. Similar interactions also occur between -OP groups of TPP with amino groups in chitosan. Thus, the TPP plays a role in the formation of densely packed nanoparticles [20].

Determination of the optimum $\mathrm{pH}$ of Au solution is important due to the acidity of the medium affects the charge of the FF-Chitosan surface and its stability. The acidity of Au solution was set at acid condition ( $\mathrm{pH}$ 1-6), where gold ion $(\mathrm{Au}(\mathrm{III}))$ has negative charge as $\mathrm{AuCl}_{4}^{-}$, $\left[\mathrm{AuCl}_{3}(\mathrm{OH})\right]^{-},\left[\mathrm{AuCl}_{2}(\mathrm{OH})_{2}\right]^{-}$, and $\left[\mathrm{AuCl}(\mathrm{OH})_{3}\right]^{-}$species $[13,21]$. The optimum $\mathrm{pH}$ of $\mathrm{Au}$ solution for FFChitosan-Au synthesized in this research has been achieved at pH 2 (as shown in Fig. 2). It can be explained that at $\mathrm{pH} \mathrm{1,} \mathrm{Au} \mathrm{that} \mathrm{binds} \mathrm{to} \mathrm{the} \mathrm{active} \mathrm{site} \mathrm{of} \mathrm{FF-}$ Chitosan was low because of the strong acidity environment leading to ion competition between $\left[\mathrm{AuCl}_{4}\right]^{-}$with $\mathrm{Cl}^{-}$of $\mathrm{HCl}$. Au ion and FF-Chitosan surface electrostatic interaction has reached the maximum at $\mathrm{pH}$ 2 and decreased slightly until $\mathrm{pH}$ 6. This happened due to the $\mathrm{H}^{+}$ion in the Au solution that protonates $-\mathrm{NH}_{2}$ and $-\mathrm{OH}$ groups in FF-Chitosan was decreased with the increase in $\mathrm{pH}$. Another reason is, at $\mathrm{pH} 5$ and $6, \mathrm{Au}$ ions have a negative charge as $\left[\mathrm{AuCl}_{2}(\mathrm{OH})_{2}\right]^{-}$and $\left[\mathrm{AuCl}(\mathrm{OH})_{3}\right]^{-}$, which have lower electrostatic attraction than at lower $\mathrm{pH}$ [13]. Since the maximum capacity of $\mathrm{Au}$ that binds to the FF-Chitosan surface occurred at $\mathrm{pH} 2$ in the examined $\mathrm{pH}$ range, the following synthesis of FFChitosan-Au was carried out at $\mathrm{pH} 2$.

The initial concentration of Au solution becomes an important driving force that affects the adsorption capacity of FF-Chitosan to Au ion. The amount of Au ion that binds to FF-Chitosan surface at $\mathrm{pH} 2$ and room temperature is shown in Fig. 3, which informs the correlation of adsorption capacity of FF-Chitosan with

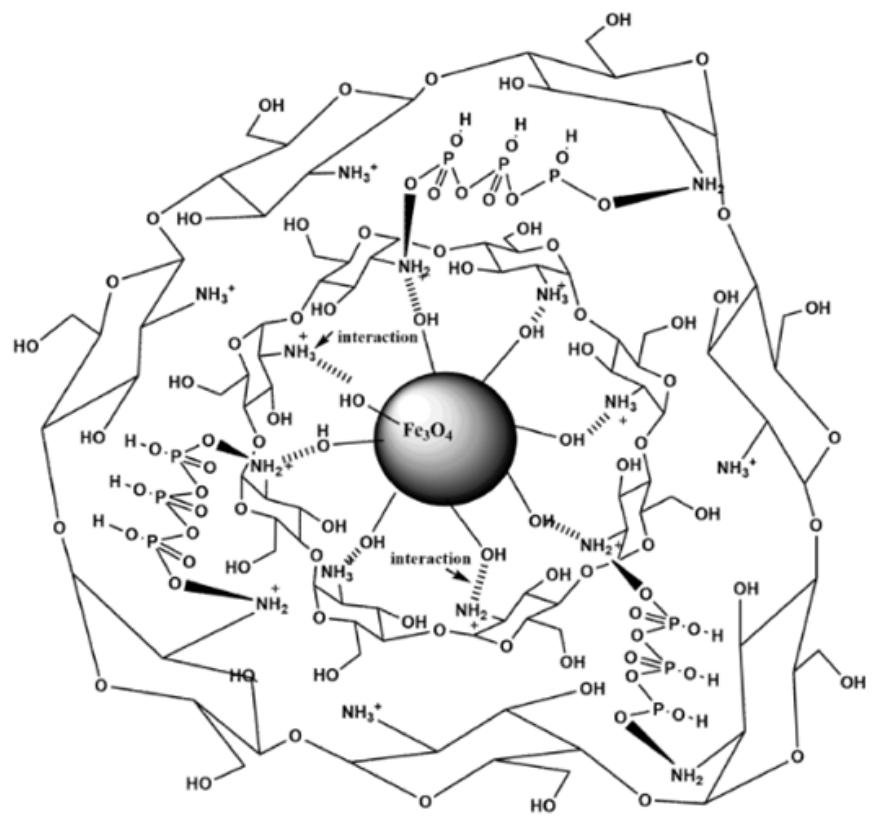

Fig 1. Schematic illustration of the interaction between $\mathrm{Fe}_{3} \mathrm{O}_{4}$ and chitosan with cross-linker TPP

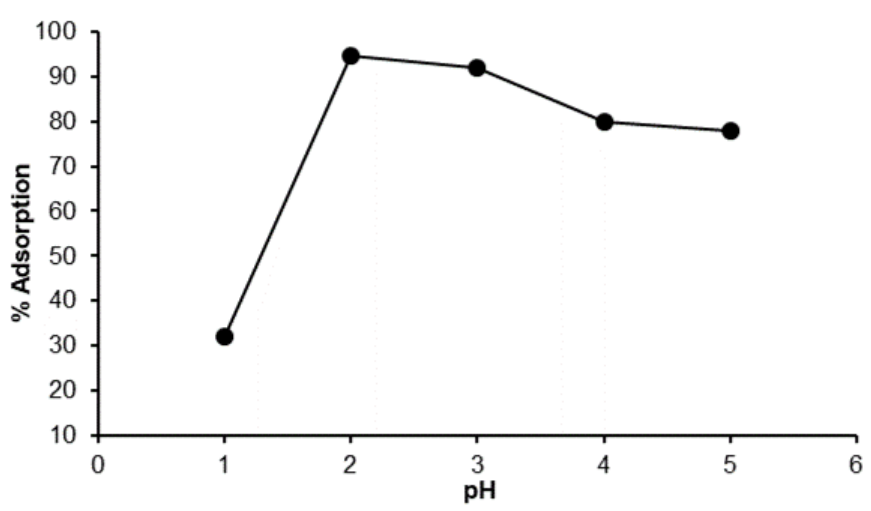

Fig 2. Determination of the optimum $\mathrm{pH}$ on the FFChitosan-Au synthesis

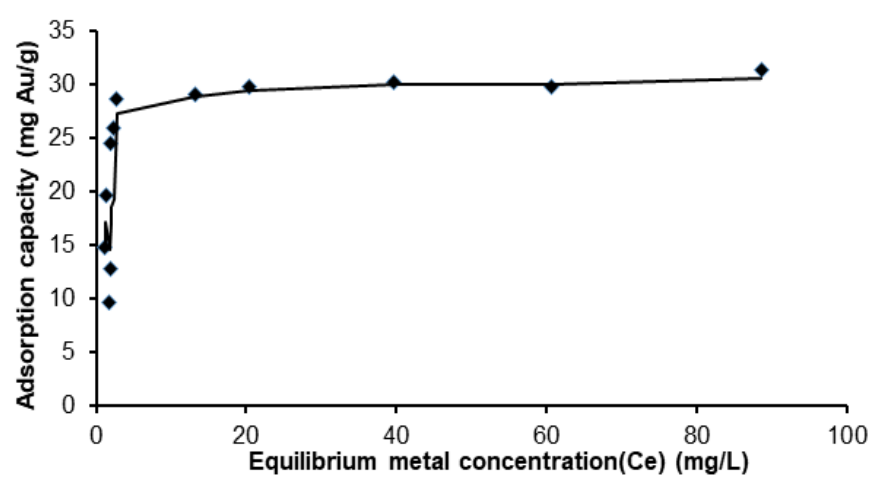

Fig 3. Equilibrium isotherm for the adsorption of $\mathrm{Au}$ ions on the FF-Chitosan in the synthesis of FF-Chitosan$\mathrm{Au}$ 
$\mathrm{Au}$ ion concentration at equilibrium $(\mathrm{Ce})$. As the initial metal ion concentration increases, the percentage of $\mathrm{Au}$ that binds to FF-Chitosan decreases, which was in line with the basic concept of mass transfer [22]. At a low concentration of $\mathrm{Au}$ ion, the active binding sites of FFChitosan remain unsaturated, offering a large surface area for $\mathrm{Au}$ ion. At a higher concentration, $\mathrm{Au}$ ion leads to a decreasing in the total surface area of the $\mathrm{Au}$ ion adsorbent [13]. After the equilibrium has been reached, the increasing concentration in the system caused the distance of particles to narrow and limited the mobility of particles, then made the amount of $\mathrm{Au}$ ion that binds to FF-Chitosan decrease. Fig. 3 informs the adsorption capacity of FF-Chitosan to Au is $30 \mathrm{mg} \mathrm{Au} / \mathrm{g}$ FF-Chitosan.

The adsorption isotherm that informs the correlation between the amount of $\mathrm{Au}$ per unit mass of FF-Chitosan at constant temperature and its concentration in the equilibrium solution is important to determine the capacity of $\mathrm{Au}$ ion that binds onto FFChitosan surface. The calculation was done by fitting the experimental data into the Langmuir and Freundlich isotherm models. Table 1 shows the coefficient correlation of the Langmuir and Freundlich models of FFChitosan to Au ion. Mass of FF-Chitosan was varied by 5 , 10 , and $15 \mathrm{mg}$ to get more accurate information.

Based on the coefficient correlation of Langmuir and Freundlich, the adsorption capacity of the FFChitosan-Au synthesis fits the Langmuir model. Langmuir adsorption isotherm is examined based on the adsorbent monolayer surface, and all of the active sites on the adsorbent surface give the same affinity to interact with adsorbate [13]. The calculation result shown in Table 2 confirms the maximum adsorption capacity and $\mathrm{Au}$ loading content of $30.24 \mathrm{mg} \mathrm{Au/g} \mathrm{FF-Chitosan.}$

Table 1. Coefficient correlation of the Langmuir and Freundlich models of the adsorption FF-Chitosan to $\mathrm{Au}$

\begin{tabular}{ccc}
\hline \multirow{2}{*}{$\begin{array}{c}\text { Mass of FF-Chitosan } \\
(\mathrm{mg})\end{array}$} & \multicolumn{2}{c}{$\mathrm{R}^{2}$} \\
\cline { 2 - 3 } & Langmuir & Freundlich \\
\hline 5 & 0.969 & 0.069 \\
10 & 0.999 & 0.465 \\
15 & 0.995 & 0.449 \\
\hline
\end{tabular}

Table 2. Langmuir parameters of FF-Chitosan-Au synthesis

\begin{tabular}{cccc}
\hline \multirow{2}{*}{$\begin{array}{c}\text { FF-Chitosan } \\
(\mathrm{mg})\end{array}$} & \multicolumn{3}{c}{ Langmuir Isotherm } \\
\cline { 2 - 4 } & $\left(\mathrm{R}^{2}\right)$ & $\begin{array}{c}\mathrm{Kl} \\
(\mathrm{L} / \mathrm{mg})\end{array}$ & $\begin{array}{c}\text { Qmax } \\
(\mathrm{mg} / \mathrm{g})\end{array}$ \\
\hline 5 & 0.969 & 0.219 & 30.77 \\
10 & 0.999 & 1.622 & 30.21 \\
15 & 0.995 & 0.938 & 29.76 \\
\hline Average & & & 30.24 \\
\hline
\end{tabular}

\section{Characterization of Materials}

FTIR spectrophotometric analysis was carried out to verify the functional groups that were present in the synthesized materials. Fig. 4 shows the functional groups of chitosan (a), FF-Chitosan (b), and FF-Chitosan-Au (c). The broad absorption band at wavenumber 3361 $\mathrm{cm}^{-1}$ attributed to $\mathrm{O}-\mathrm{H}$ and $\mathrm{N}-\mathrm{H}$ vibrations (Fig. $4 \mathrm{a}-\mathrm{c}$ ). Characteristic of chitosan peak shown at $2926 \mathrm{~cm}^{-1}$ (C-H stretching), $1648 \mathrm{~cm}^{-1}$ (N-H stretching), $1378 \mathrm{~cm}^{-1}$ (C-C bending), $1315 \mathrm{~cm}^{-1}$ (C-N stretching), $1071 \mathrm{~cm}^{-1}$ (C-O asymmetry), $1035 \mathrm{~cm}^{-1}$ (C-O symmetry) and $894 \mathrm{~cm}^{-1}$ (C-H ring stretching) [10]. FTIR spectra FFChitosan (Fig. 4(b)) also show $\mathrm{O}-\mathrm{H}$ and $\mathrm{N}-\mathrm{H}$ absorption peaks at $3363 \mathrm{~cm}^{-1}$. The peak at $1648 \mathrm{~cm}^{-1}$ belongs to the stretching vibration of $\mathrm{N}-\mathrm{H}$ from chitosan. The new peak at $582 \mathrm{~cm}^{-1}$ (Fig. 4(b)) can be related to the stretching vibration of $\mathrm{Fe}-\mathrm{O}$ from $\mathrm{Fe}_{3} \mathrm{O}_{4}$ in FF-Chitosan [7,22]. The absorption bands at $455 \mathrm{~cm}^{-1}$ attributed to tetrahedral and octahedral sites of $\mathrm{Fe}_{3} \mathrm{O}_{4}$ [23]. The enhanced peak at $582 \mathrm{~cm}^{-1}$ at Fig. 4(c) suggested that FF-Chitosan-Au already formed successfully [24].

Diffraction analysis of the samples has been done to confirm the existence of $\mathrm{Fe}_{3} \mathrm{O}_{4}$ and $\mathrm{Au}$ in the FFChitosan and FF-Chitosan-Au. Diffractogram of FFChitosan in Fig. 5(a) shows the seven characteristic peaks of $\mathrm{Fe}_{3} \mathrm{O}_{4}$ at $30.26^{\circ}, 35.49^{\circ}, 43.24^{\circ}, 53.63^{\circ}, 57.14^{\circ}$, $62.81^{\circ}, 74.38^{\circ}$ [25]. The peak of chitosan was observed at $2 \theta=20.0^{\circ}$ with low intensity and wide bump, indicating the amorphous phase [13]. The XRD result of FFChitosan-Au (Fig. 5(b)) shows some characteristic peaks that also appear at the FF-Chitosan diffractogram, which 


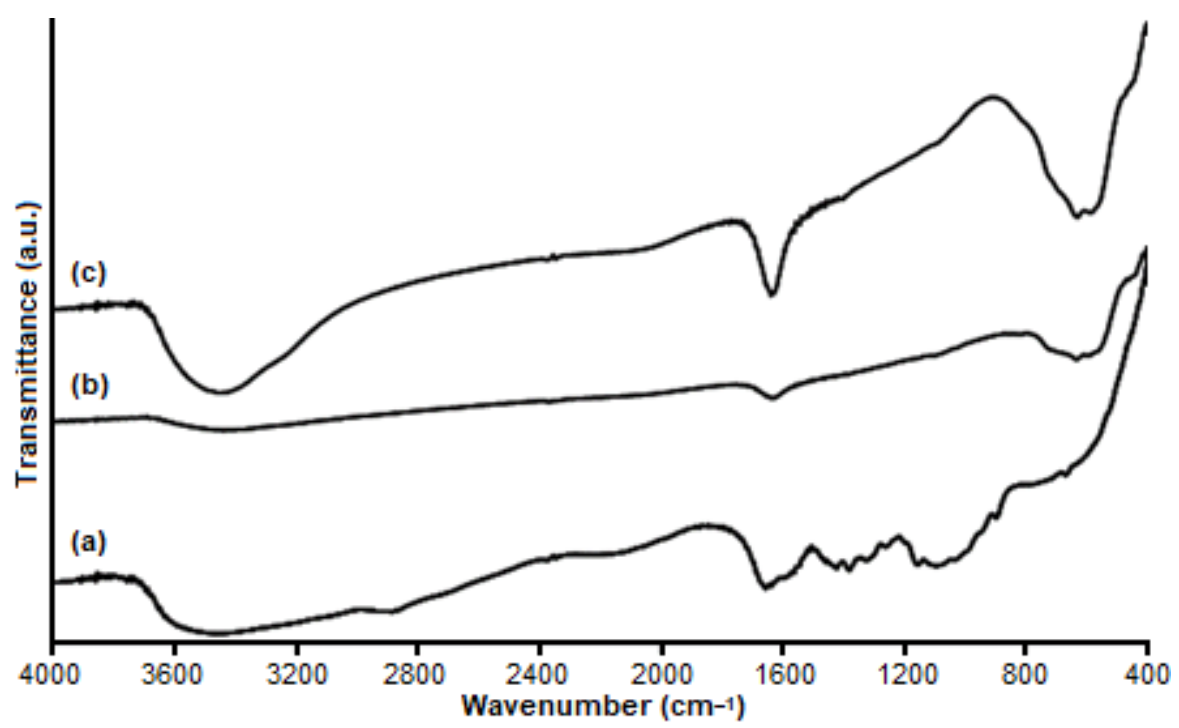

Fig 4. FTIR spectra of (a) Chitosan, (b) FF-Chitosan and (c) FF-Chitosan-Au

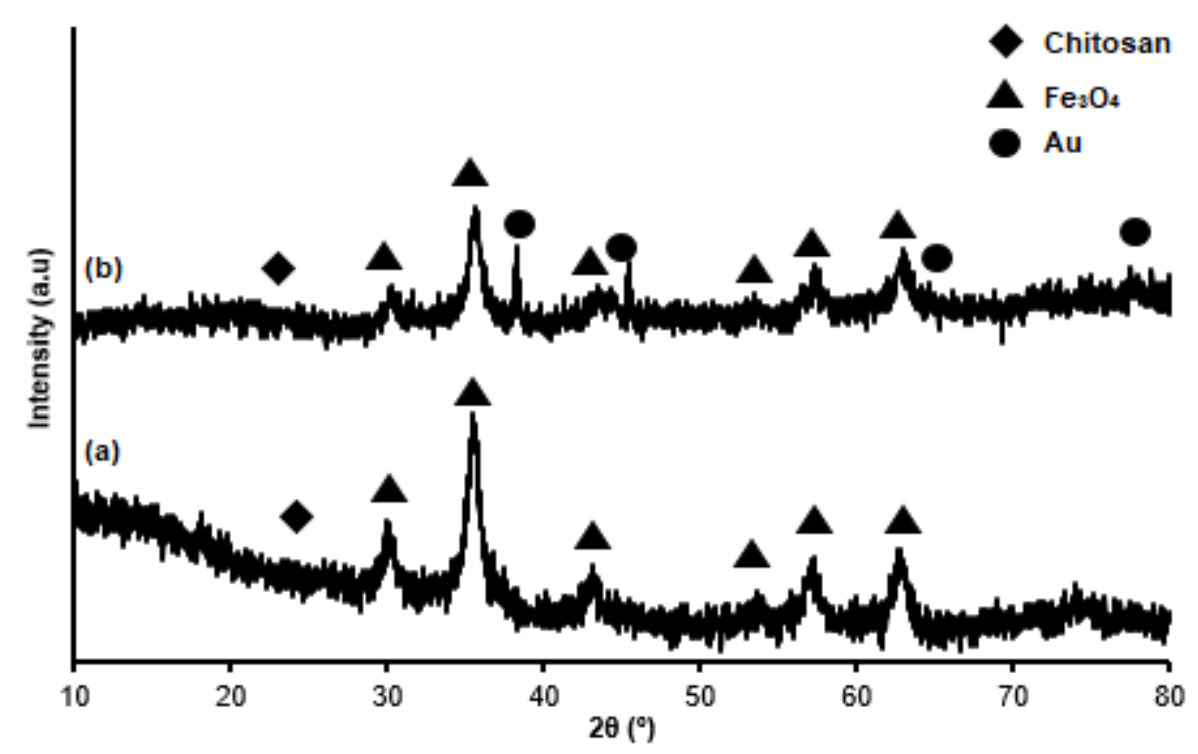

Fig 5. Diffraction pattern of (a) FF-Chitosan and (b) FF-Chitosan-Au

belong to $\mathrm{Fe}_{3} \mathrm{O}_{4}$ and chitosan. Other peaks also appear that belong to $\mathrm{Au}$ at $2 \theta 37.94^{\circ}, 44.13^{\circ}, 64.22^{\circ}$, and $77.03^{\circ}$ [26]. Peaks of $\mathrm{Au}$ corresponds to COD-Inorg entry number 96-901-3039. It indicates that $\mathrm{Au}$ has embedded at FF-Chitosan [23].

Particle size distribution of FF-Chitosan with dynamic light scattering (DLS) method presented in Fig. 6 shows a narrow distribution particle size with an average size of $90.57 \mathrm{~nm}$ with a polydispersity index (PDI) of 0.150 . The value of the polydispersity index indicates the homogeneity of FF-Chitosan particles is polydisperse (if the PDI $<0.1$, the particle distribution is monodispersed). In drug delivery applications, a PDI value of 0.3 or below is acceptable. Previous research on coating magnetic nanoparticles using chitosan has broad particle distribution (20-100 nm) [25]. However, the polydispersity of the samples is low (homogeneity of the sample is high as confirmed with SEM). The particle size distribution of FF-Chitosan-Au also shows narrow distribution particle size in the nanometer range (average size $82.93 \mathrm{~nm}$ ) with a polydispersity index of 0.175 , indicating a good homogeneity. A self-assembly 

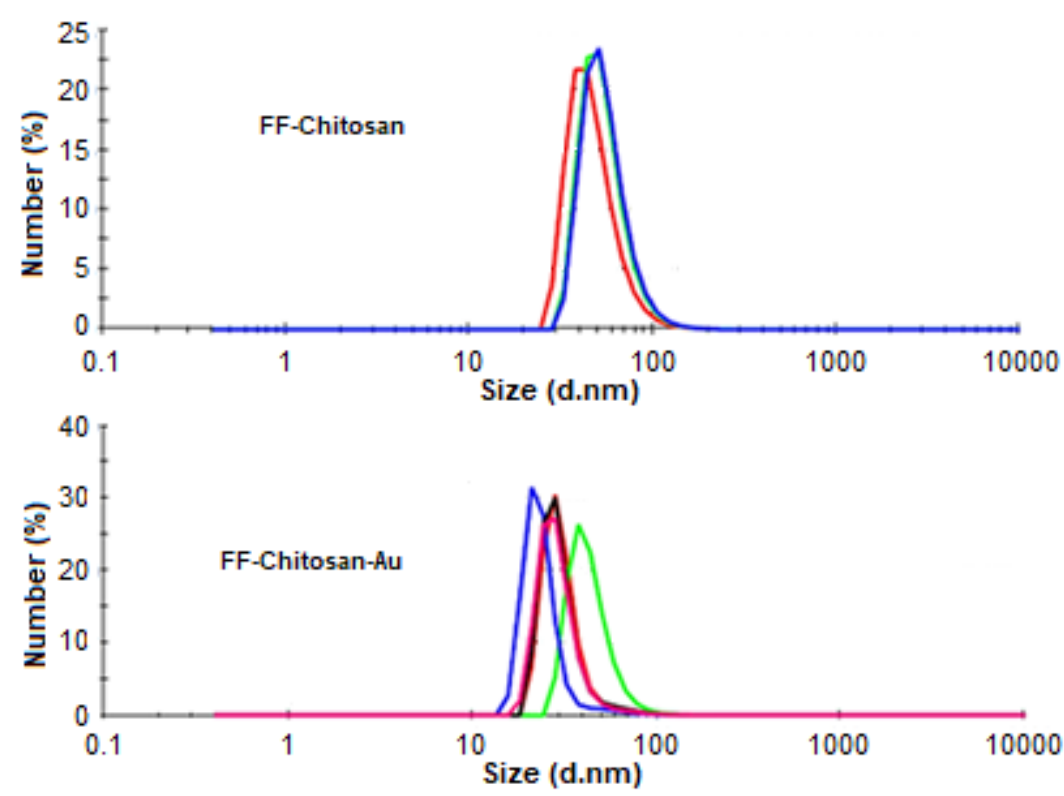

Fig 6. Particle size distribution of FF-Chitosan and FF-Chitosan-Au

process of $\mathrm{Au}$ to FF-Chitosan surface may cause a reduction of the tendency of magnetic chitosan to agglomerate (magnetic interaction), making slight changes in the increasing of the polydispersity index, decreasing the average size.

The morphology of the prepared nanoparticles was examined using SEM, as shown in Fig. 7. SEM micrograph of nanoparticles FF-Chitosan indicates that the particles have a spherical shape and good uniformity in size with a diameter less than $100 \mathrm{~nm}$ (Fig. 7(a)). This result corresponds to the FF-Chitosan particle size obtained from the DLS method that the polydispersity index of FFChitosan is 0.1 , which is close to monodisperse $(<0.1)$. In the FF-Chitosan in SEM micrograph seemed to form clusters, but still in nanometers size, due to intermolecular interactions, such as electrostatic, hydrophobic, magnetic, and van der Waals interactions during sample preparation in SEM analysis. SEM micrograph of FF-Chitosan-Au (Fig. 7(b)) shows that FF-Chitosan-Au has a spherical shape with homogeneous size distribution. There are also several nanoparticles agglomeration but still in the nanometer range.

Magnetic properties of the samples have been characterized using Vibrating Sample Magnetometer (VSM) and presented in a hysteresis curve. Fig. 8 shows
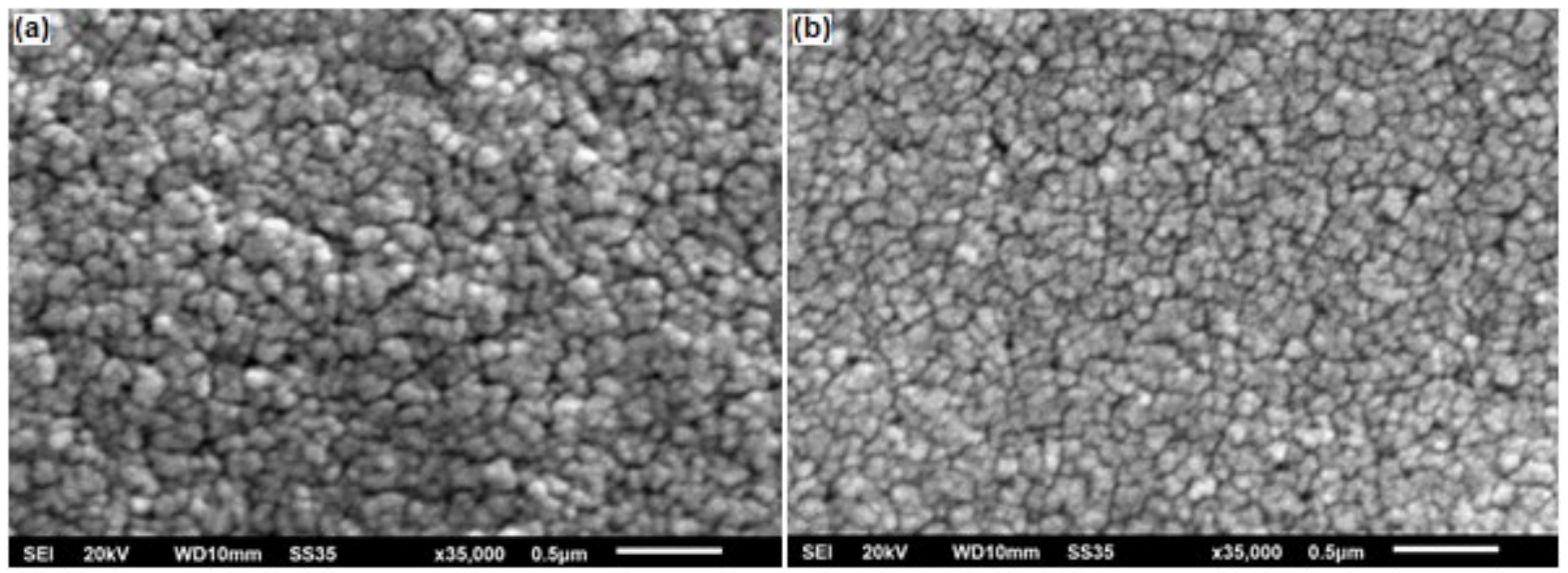

Fig 7. Scanning electron micrograph (SEM) image of FF-Chitosan (a) and FF-Chitosan-Au (b) 


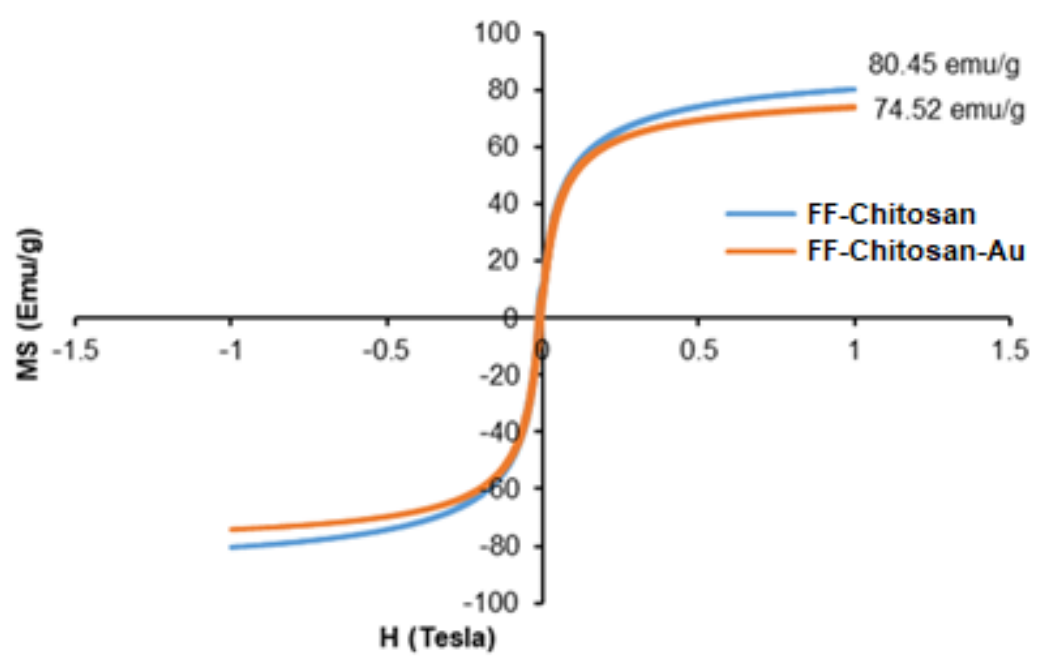

Fig 8. Hysteresis curve of FF-Chitosan and FF-Chitosan-Au

a hysteresis curve of FF-Chitosan and FF-Chitosan-Au, respectively. Base on particle size data from the DLS method and SEM, the size of FF-Chitosan and FFChitosan- $\mathrm{Au}$ in the tens nanometers show superparamagnetic behavior. The magnetization of superparamagnetic nanoparticles will be zero in the absence of an external magnetic field, while the coercivity and remanence will be nearly zero in its hysteresis curve [6]. Fig. 8 shows that the magnetization saturation (Ms) value of FF-Chitosan is $80.45 \mathrm{emu} / \mathrm{g}$. Compared to another magnetic-chitosan research [22,27], the Ms value in this research is higher. The Ms value of FF-Chitosan$\mathrm{Au}$ is $74.52 \mathrm{emu} / \mathrm{g}$, which lower than FF-Chitosan because $\mathrm{Au}$ has diamagnetic properties that hamper magnetic interaction between magnetic nanoparticles. This also indicates that Au has been embedded in FF-Chitosan.

\section{- CONCLUSION}

This study reports the synthesis of FerrofluidChitosan-Au in two steps procedure. FF-Chitosan was synthesized through in-situ co-precipitation, then Au was assembled to FF-Chitosan via adsorption process. The maximum capacity of Au embedded in FF-Chitosan is 30 $\mathrm{mg} \mathrm{Au/g} \mathrm{FF-Chitosan} \mathrm{and} \mathrm{fits} \mathrm{the} \mathrm{Langmuir} \mathrm{isotherm}$ model. Characterization results from FTIR, SEM, XRD show that FF-Chitosan-Au has been formed in the nanometer range with a $\mathrm{Ms}$ value of $74.52 \mathrm{emu} / \mathrm{g}$. Considering its effectiveness and safety synthesis route, this FF-Chitosan-Au synthesis procedure could be employed when using $\mathrm{Au}$ in an active phase, promisingly in a biomedical application (brachytherapy agent).

\section{- ACKNOWLEDGMENTS}

We acknowledge the financial support from the research funding DIPA 2018. We also would like to thank Mr. Ajrieh Setyawan from PTLR-BATAN for his assistance with the FAAS analysis.

\section{- REFERENCES}

[1] Pati, S.S., Singh L.H., Oliveira, A.C., and Garg, V.K., 2016, Chitosan functionalized $\mathrm{Fe}_{3} \mathrm{O}_{4} @ A$ u core-shell nanomaterials for targeted drug delivery, Int. J. Chem. Mol. Nucl. Mater. Metall. Eng., 9 (6), 643-646.

[2] Pati, S.S., Singh, L.H., Guimar, E.M., Mantilla, J., Coaquira, J.A.H., Oliveira, A.C., Sharma, V.K., and Garg, V.K., 2016, Magnetic chitosan-functionalized $\mathrm{Fe}_{3} \mathrm{O}_{4} @ \mathrm{Au}$ nanoparticles: Synthesis and characterization, J. Alloys Compd., 684, 68-74.

[3] Sun, S.N., Wei, C., Zhu, Z.Z., Hou, Y.L., Venkatraman, S.S., and Xu, Z.C., 2014, Magnetic iron oxide nanoparticles: Synthesis and surface coating techniques for biomedical applications, Chin. Phys. B, 23 (3), 037503.

[4] Leamy, P.J., 2003, Preparation, characterization, and in vitro testing of poly(lactide-co-glycolide) and dextran magnetic microspheres for in vivo applications, Dissertation, University of Florida. 
[5] Mohammed, L., 2016, Polymeric superparamagnetic nanoparticles for drug delivery applications, Thesis, University of Western Ontario.

[6] Sulungbudi, G.T., and Mujamilah, 2016, An effort on homogenizing size distribution of magnetic nanosphere $\mathrm{Fe}_{3} \mathrm{O}_{4}$-poly-lactic acid for hyperthermia treatment, $M P I, 19$ (1), 68-83.

[7] Ebrahimi, M., 2016, A short review on ferrofluids surface modification by natural and biocompatible polymers, Nanomed. J., 3 (3), 155-158.

[8] Wu, W., He, Q., and Jiang, C., 2008, Magnetic iron oxide nanoparticles: Synthesis and surface functionalization strategies, Nanoscale Res. Lett., 3 (11), 397.

[9] Mahdavi, M., Ahmad, M.B., Haron, J., Namvar, F., Nadi, B., Rahman, M.Z., and Amin, J., 2013, Synthesis, surface modification and characterisation of biocompatible magnetic iron oxide nanoparticles for biomedical applications, Molecules, 18 (7), 75337548.

[10] Pylypchuk, I.V., Kołodyńska, D., Kozioł, M., and Gorbyk, P.P., 2016, Gd-DTPA adsorption on chitosan/magnetite nanocomposites, Nanoscale Res. Lett., 11 (1), 168.

[11] Qu, H., Ma, H., Riviere, A., Zhou, W., and Connor, C.J.O., 2012, One-pot synthesis in polyamines for preparation of water-soluble magnetite nanoparticles with amine surface reactivity, J. Mater. Chem., 22 (8), 3311-3313.

[12] Abouelmagd, S.A., Ku, Y.J., and Yeo, Y., 2015, Low molecular weight chitosan-coated polymeric nanoparticles for sustained and ph-sensitive delivery of paclitaxel, J. Drug Targeting, 23 (7-8), 725-735.

[13] Muflikhah, Rusdiarso, B., Putra, E.G.R., and Nuryono, 2017, Modification of silica coated on iron sand magnetic material with chitosan for adsorption of $\mathrm{Au}(\mathrm{III})$, Indones. J. Chem., 17 (2), 264-273.

[14] Yao, C., Zhang, L., Wang, J., He, Y., Xin, J., Wang, S., $\mathrm{Xu}, \mathrm{H}$., and Zhang, Z., 2016, Gold nanoparticle mediated phototherapy for cancer, J. Nanomater., 2016, 5497136.

[15] Boyles, M.S.P., Kristl, T., Andosch, A., Zimmermann, M., Tran, N., Casals, E., Himly, M.,
Puntes, V., Huber, C.G., Lütz-Meindl, U., and Duschl, A., 2015, Chitosan functionalisation of gold nanoparticles encourages particle uptake and induces cytotoxicity and pro-inflammatory conditions in phagocytic cells, as well as enhancing particle interactions with serum components, $J$. Nanobiotechnology, 13 (1), 84.

[16] Chanda, N., Kan, P., Watkinson, L.D., Shukla, R., Zambre, A., Carmack, T.L., Engelbrecht, H., Lever, J.R., Katti, K., Fent, G.M., Casteel, S.W., Smith, C.J., Miller, W.H., Jurisson, S., Boote, E., Robertson, J.D., Cutler, C., Dobrovolskaia, M., Kannan, R., and Katti, K.V., 2010, Radioactive gold nanoparticles in cancer therapy: Therapeutic efficacy studies of Ga${ }^{198}$ AuNP nanoconstruct in prostate tumor-bearing mice, Nanomed. Nanotechnol. Biol. Med., 6 (2), 201-209.

[17] Ritawidya, R., Pujiyanto, A., Setiawan, H., Ramli, M., and Kurniasih, D., 2012, Synthesis and characterization of poly(amidoamine) dendrimers encapsulated ${ }^{198} \mathrm{Au}$ nanoparticles, At. Indones., 38 (3), 118-126.

[18] Hemalatha, T., Prabu, P., Gunadharini, D.N., and Gowthaman, M.K., 2018, Fabrication and characterization of dual acting oleyl chitosan functionalised iron oxide/gold hybrid nanoparticles for MRI and CT imaging, Int. J. Biol. Macromol., 112, 250-257.

[19] Nasution, T.I., Asrosa, R., Nainggolan, I., Balyan, M., Indah, R., and Wahyudi, A., 2018, Sodium tripolyphosphate cross-linked chitosan based sensor for enhancing sensing properties towards acetone, IOP Conf. Ser.: Mater. Sci. Eng., 309, 012083.

[20] Unsoy, G., Yalcin, S., Khodadust, R., Gunduz, G., and Gunduz, U., 2012, Synthesis optimization and characterization of chitosan-coated iron oxide nanoparticles produced for biomedical applications, J. Nanopart. Res., 14 (11), 964.

[21] Parisien-La Salle, J.C., 2012, In-situ adsorption of gold(III) chloride on activated carbon under pressure oxidation-leaching conditions, Thesis, Mc Gill University. 
[22] Zhao, M., Zhao, J., Huang, Z., Wang, S., and Zhang, L., 2019, One pot preparation of magnetic chitosancystamine composites for selective recovery of $\mathrm{Au}$ (III) from the aqueous solution, Int. J. Biol. Macromol., 137, 721-731.

[23] Liu, X.L., Yang, X., Xin, H.Y., Tang, X.P., Weng, L.J., Han, Y.Y., and Geng, D., 2016, Ecofriendly fabrication of $\mathrm{Au} / \mathrm{Fe}_{3} \mathrm{O}_{4}$-chitosan composites for catalytic reduction of methyl orange, Dig. J. Nanomater. Biostruct., 11 (2), 337-348.

[24] Pham, X.N., Nguyen, T.P., Pham, T.N., Tran, T.T.N., and Tran, T.V.T., 2016, Synthesis and characterization of chitosan- coated magnetite nanoparticles and their application in curcumin drug delivery, $A d v$. Nat. Sci.: Nanosci. Nanotechnol., 7 (4), 045010.
[25] Shirazi, H., Daneshpour, M., Kashanian, S., and Omidfar, K., 2015, Synthesis, characterization and in vitro biocompatibility study of $\mathrm{Au} / \mathrm{TMC} / \mathrm{Fe}_{3} \mathrm{O}_{4}$ nanocomposites as a promising, nontoxic system for biomedical applications, Beilstein J. Nanotechnol., 6, 1677-1689.

[26] Pestov, A., Nazirov, A., Modin, E., Mironenko, A., and Bratskaya, S., 2014, Mechanism of $\mathrm{Au}$ (III) reduction by chitosan: Comprehensive study with ${ }^{13} \mathrm{C}$ and ${ }^{1} \mathrm{H}$ NMR analysis of chitosan degradation products, Carbohydr. Polym., 117, 70-77.

[27] Podrepšek, G.H., Knez, Ž., and Leitgeb, M., 2020, Development of chitosan functionalized magnetic nanoparticles with bioactive compounds, Nanomaterials, 10 (10), 1913. 